\title{
A Child with Submandibular Fistulized Mass: An Unusual Case of
} Salmonella typhi

\author{
Abdollah Karimi ${ }^{1}$, Zahra Pourmoghaddas ${ }^{1,}{ }^{*}$, Arefeh Fayazi ${ }^{1}$ and Farid Imanzadeh ${ }^{2}$ \\ ${ }^{1}$ Pediatric Infections Research Center, Research Institute for Children Health, Shahid Beheshti University of Medical Sciences, Tehran, Iran \\ ${ }^{2}$ Pediatric Gastroenterology, Hepatology and Nutrition Research Center, Research Institute for Children Health, Shahid Beheshti University of Medical Sciences, Tehran, Iran \\ "Corresponding author: Pediatric Infections Research Center, Research Institute for Children Health, Shahid Beheshti University of Medical Sciences, Tehran, Iran. Email: \\ zahrapormoghadas@yahoo.com
}

Received 2018 July 23; Revised 2019 June 15; Accepted 2019 June 23.

\begin{abstract}
Salmonella typhi is a Gram-negative microorganism. This enteric pathogen causes a variety of signs and symptoms, but the more prevalent is fever and gastrointestinal signs. It is important to consider this microorganism in the differential diagnosis in patients coming from the regions with a high prevalence of salmonella typhi and unusual signs and symptoms.
\end{abstract}

Keywords: Salmonella, Lymphadenopathy, Child

\section{Introduction}

Salmonella typhi is one of the causes of enteric fever. Infected patients mostly experience fever and gastrointestinal symptoms due to the intestinal invasion of the microorganism. Zahedan is one of the regions in Iran with a high prevalence of $S$. typhi infection. This infection can present unusual signs and symptoms leading to misdiagnosis, especially in patients with immunodeficiency. The current study reported a pediatric case inflicted with $S$. typhi infection accompanied by rare and unusual signs and symptoms such as palpable petechiae on lower limbs and submandibular fistulized mass.

\section{Case Presentation}

A five-year-old Baloch female patient from Zahedan, Iran was admitted to Mofid Children Hospital due to abdominal pain, fever, and fistulized mass in submandibular area as suppurative lymphadenopathy. Her medical and developmental history was normal. No history of weight loss and chronic or recent diarrhea or constipation was reported.

She had been well until approximately six months before the recent admission. The disease started with fever, purpuric rashes, and abdominal pain primitively treated for Henoch-Schonlein purpura. The purpuric rashes and the abdominal pain were not improved after two weeks.
Therefore, skin biopsy was obtained and the leukocytoclastic vasculitis was determined as the final diagnosis. Based on the diagnosis, she was treated with immunomodulatory agents such as azathioprine and prednisolone. During this admission, ceftriaxone was administered because of fever until the blood culture became negative.

Two months later, she was admitted again due to fever and severe abdominal pain, mimicking acute abdominal manifestations. Her family complained about waxing and waning of her gastrointestinal discomfort during the last two months. Parents declared that during the last month family physician administered cefixime for her 2 times because of abdominal pain and fever. This time the appendectomy was performed since pathological reports showed appendicitis and necrosis.

The physical examinations showed some enlarged lymph nodes with prominent lymphadenitis. Moreover, her purpuric rashes were present, but with less severity than the previous admission. The immune-suppressive therapy was continued, according to the pediatric rheumatologist prescription. Four months later, she came back again with abdominal pain, fistulized draining mass in the submandibular area (Figure 1) and fever. Only the tenderness of the right lower quadrant was prominent in the physical examination. Abdominal sonography revealed several accumulated lymphadenopathies, some of which were necrotic with a maximum dimension of $33 \times 15 \mathrm{~mm}$ in the para-aortic region, along with inflammatory evidence such as increased echogenicity in the mesenteric fat 


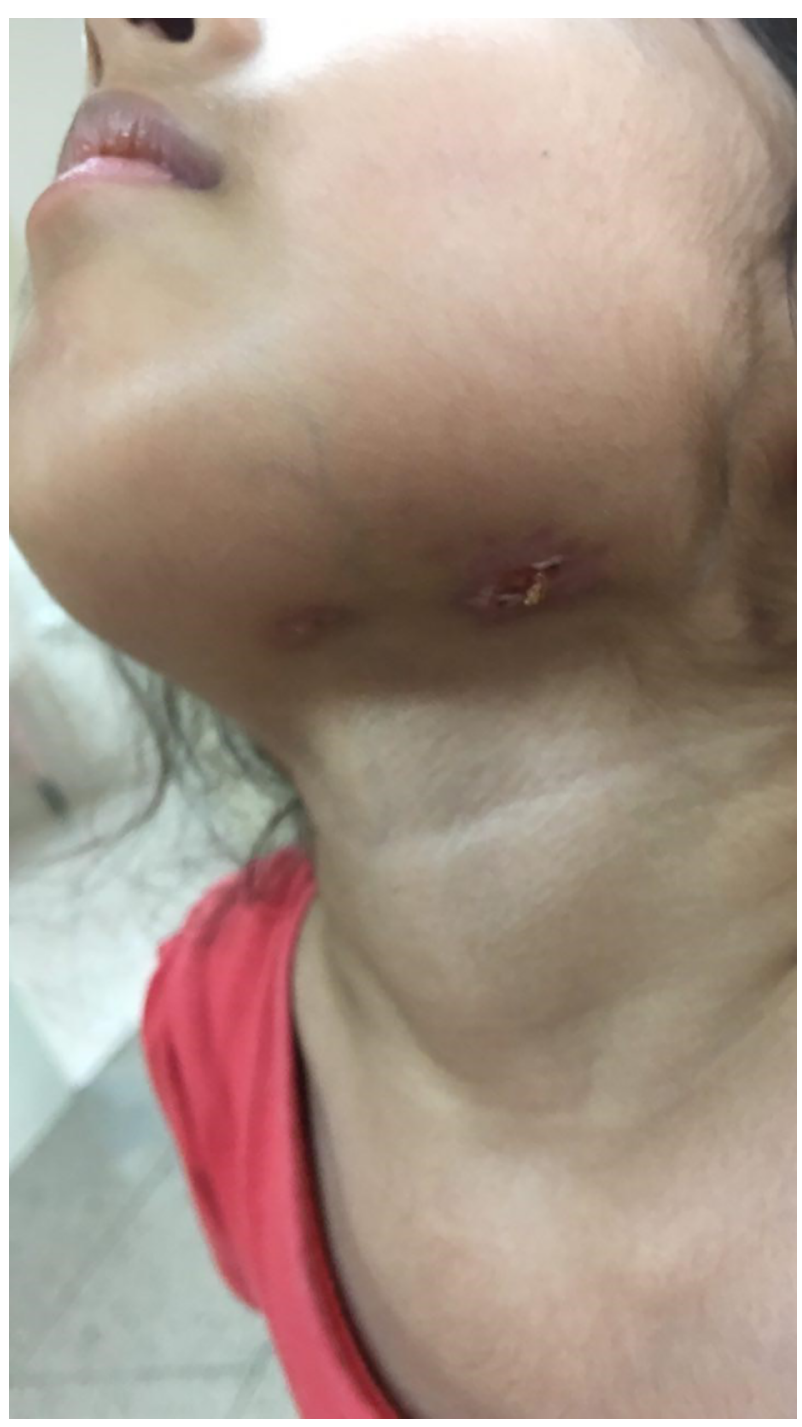

Figure 1. Draining lymph node is shown.

and right lower quadrant.

Therefore, a consultation was made with a pediatric infectious disease specialist. Tuberculosis (TB) or other inflammatory conditions such as inflammatory bowel disease (IBD) were among the differential diagnoses. In addition, the patient was evaluated for brucellosis and salmonellosis. Among all assessments, the blood, urine, stool, and wound cultures were positive for Salmonella typhi.

Normal results were obtained in the evaluation of other organs such as heart, eye, and immune system (Table 1). The patient was treated for disseminated salmonellosis. The immune-suppressive therapy was stopped since

\begin{tabular}{lc}
\hline Table 1. The Immune System Screening Tests & \\
\hline Variables & Values \\
\hline IgA, mg/mL & 122 \\
\hline IgG, mg/mL & 678 \\
\hline IgM, mg/mL & 68 \\
\hline CD4,\% & 35 \\
\hline CD8, \% & 25 \\
\hline CD19, \% & 15 \\
\hline CD16/56, \% & 11.7 \\
\hline WBC & 15500 \\
\hline Neut, \% & 80 \\
\hline Lymph, \% & 18 \\
\hline
\end{tabular}

all signs and symptoms from the first time of admission were attributed to salmonellosis. After the administration of antibiotic agents (based on the antibiogram results), a dramatic response was observed. Fever disappeared after 24 hours, lymphadenitis was resolved, and the obvious cure of the general condition was achieved within 15 days of treatment.

\section{Discussion}

Enteric fever is a systemic disease caused by S. typhi with an annual prevalence of 12-13 million cases mainly in Southeast Asia, where poor sanitation and the lack of safe drinking water are mostly accused (1-3). Gastrointestinal signs and symptoms such as acute abdominal pain, vomiting, and diarrhea are the most common manifestations of this condition $(2,4)$.

Rose spots are the characteristic skin lesions of typhoid fever (5). Other skin rashes such as purpura are rare and only reported in patients with endocarditis (6). A study reported a typhoid fever case with leukocytoclastic purpura without endocarditis (7). In other reports, it was believed that this kind of vasculitis is associated with typhoid fever with an immunodeficiency condition such as mutations in the IL-12R $\beta 1$ gene $(8,9)$.

The atypical presentation of salmonellosis is usually reported in infants and the elderly. Malnutrition and chronic illnesses are the predisposing factors of uncommon presentation of this infection in different case reports (10). It is documented that cellular immunity is essential for killing Salmonella spp., so the atypical presentation and dissemination of this infection is expected in patients with immunodeficiency and cellular immunity involvements (9).

The involvement of cervical lymph nodes in salmonellosis may be due to the direct invasion of tonsillar 
lymph nodes or hematogenous invasion by this Gramnegative bacillus. In the present patient, it seems that the hematogenous invasion was the main reason for submandibular lymph nodes involvement.

Patients with mutations in the IL-12R $\beta 1$ gene are extremely susceptible to infections with intracellular pathogens such as environmental non-tuberculous mycobacteria (NTM), Salmonella species, and even BCG vaccines (11-15). In this group of patients, lymphadenopathy is mostly recorded at the time of infection (16). The present patient was screened for immunodeficiency, but the results of all evaluations were normal; however, the evaluation of the IL-12R $\beta 1$ gene mutation was unavailable in the region and this test is pending. Nevertheless, after 12 months of follow-up without treatment with antibiotics and interferon-gamma, she presented with no signs and symptoms. On the other hand, this child took antibiotics because of wax and wane of signs and symptoms, but the courses were not enough for her illnesses and the dissemination of the infection happened due to immunosuppressive agents.

\subsection{Conclusions}

It is important to consider salmonellosis in patients from high prevalence regions, presenting unusual manifestations such as leukocytoclastic vasculitis, prolonged fever, and abdominal pain.

\section{Footnotes}

Conflict of Interests: There is no conflict of interest. Funding/Support: It is not declared by the authors.

Patient Consent: Parents in written consent let us explain the child history without the name and face of their child.

\section{References}

1. Kumar S, Rizvi M, Berry N. Rising prevalence of enteric fever due to multidrug-resistant Salmonella: An epidemiological study. J Med Microbiol. 2008;57(Pt 10):1247-50. doi: 10.1099/jmm.0.2008/001719-0. [PubMed: 18809553].

2. Crump JA, Mintz ED. Global trends in typhoid and paratyphoid Fever. Clin Infect Dis. 2010;50(2):241-6. doi: 10.1086/649541. [PubMed: 20014951]. [PubMed Central: PMC2798017].
3. Kanungo S, Dutta S, Sur D. Epidemiology of typhoid and paratyphoid fever in India.JInfect Dev Ctries. 2008;2(6):454-60. doi:10.3855/jidc.161. [PubMed: 19745523].

4. Wattiau P, Boland C, Bertrand S. Methodologies for Salmonella enterica subsp. enterica subtyping: Gold standards and alternatives. Appl Environ Microbiol. 2011;77(22):7877-85. doi: 10.1128/AEM.05527-11. [PubMed: 21856826]. [PubMed Central: PMC3209009].

5. Miller SI. Salmonella (including Salmonella typhi). Principles and practices of infectious diseases. 1995. p. 2013-33.

6. Cohen JI, Bartlett JA, Corey GR. Extra-intestinal manifestations of salmonella infections. Medicine (Baltimore). 1987;66(5):349-88. doi: 10.1097/00005792-198709000-00003. [PubMed: 3306260].

7. Shiari R, Mirjavadi S, Parvane V, Yegane M. Leukocytoclastic vasculitis associated with typhoid fever. Ann Pediatr Rheumatol.2012;1(4):240. doi: 10.5455/apr.111720120603.

8. Filiz S, Kocacik Uygun DF, Verhard EM, van Dissel JT, Uygun V, Bassorgun C, et al. Cutaneous leukocytoclastic vasculitis due to Salmonella enteritidis in a child with interleukin-12 receptor beta1 deficiency. Pediatr Dermatol. 2014;31(2):236-40. doi: 10.1111/j.15251470.2012.01856.x. [PubMed: 23004925].

9. Bustamante J, Boisson-Dupuis S, Abel L, Casanova JL. Mendelian susceptibility to mycobacterial disease: Genetic, immunological, and clinical features of inborn errors of IFN-gamma immunity. Semin Immunol. 2014;26(6):454-70. doi: 10.1016/j.smim.2014.09.008. [PubMed: 25453225]. [PubMed Central: PMC4357480].

10. Ahmed N, Saeed ZI, Tariq M. Abdominal lymphadenopathy: an atypical presentation of enteric fever. Asian Pac J Trop Biomed. 2012;2(5):409-10. doi: 10.1016/S2221-1691(12)60066-5. [PubMed: 23569940]. [PubMed Central: PMC3609307].

11. de Jong R, Altare F, Haagen IA, Elferink DG, Boer T, van Breda Vriesman PJ, et al. Severe mycobacterial and Salmonella infections in interleukin-12 receptor-deficient patients. Science. 1998;280(5368):1435-8. doi: 10.1126/science.280.5368.1435. [PubMed: 9603733].

12. Altare F, Durandy A, Lammas D, Emile JF, Lamhamedi S, Le Deist F, et al. Impairment of mycobacterial immunity in human interleukin12 receptor deficiency. Science. 1998;280(5368):1432-5. doi: 10.1126/science.280.5368.1432. [PubMed: 9603732].

13. Lammas DA, Casanova JL, Kumararatne DS. Clinical consequences of defects in the IL-12-dependent interferon-gamma (IFN-gamma) pathway. Clin Exp Immunol. 2000;121(3):417-25. doi: 10.1046/j.1365-2249.2000.01284.x. [PubMed: 10971505]. [PubMed Central: PMC1905729].

14. Staretz-Haham O, Melamed R, Lifshitz M, Porat N, Fieschi C, Casanova $\mathrm{JL}$, et al. Interleukin-12 receptor beta1 deficiency presenting as recurrent Salmonella infection. Clin Infect Dis. 2003;37(1):137-40. doi: 10.1086/375229. [PubMed: 12830418 ].

15. Remus N, Reichenbach J, Picard C, Rietschel C, Wood P, Lammas D, et al. Impaired interferon gamma-mediated immunity and susceptibility to mycobacterial infection in childhood. Pediatr Res. 2001;50(1):813. doi: 10.1203/00006450-200107000-00005. [PubMed: 11420412].

16. Dorman SE, Holland SM. Interferon-gamma and interleukin-12 pathway defects and human disease. Cytokine Growth Factor Rev. 2000;11(4):321-33. [PubMed: 10959079]. 